\title{
ON CERTAIN GROUPS OF CENTRAL TYPE
}

\author{
ALBERTO ESPUELAS
}

\begin{abstract}
A finite group $G$ is a group of central type if there exists $\chi \in$ $\operatorname{Irr}(G)$ with $\chi(1)^{2}=|G: Z(G)|$. It is known that, in such conditions, $G$ is solvable. Here some conditions assuring the nilpotence of groups of central type are given.
\end{abstract}

Let $G$ be a finite group, $N$ a normal subgroup of $G$ and $\theta \in \operatorname{Irr}(N)$ invariant in $G$. Then we say that $(G, N, \theta)$ is a character triple. Such a triple is fully ramified provided that one of the following (equivalent) conditions holds:

(i) If $\chi \in \operatorname{Irr}(G \mid \theta)$ then $\chi$ vanishes off $N$.

(ii) $\theta^{G}$ has a unique irreducible constituent $\chi$.

(iii) If $\chi \in \operatorname{Irr}(G \mid \theta)$ then $(\chi(1) / \theta(1))^{2}=|G: N|$ (see [5, p. 95]).

We say that $\chi$ is fully ramified in $N$ and $\theta$ is fully ramified in $G$. A group $G$ is of central type provided that there exists $\chi \in \operatorname{Irr}(G)$ with $\chi(1)^{2}=|G: Z(G)|$. If $\lambda$ is the irreducible constituent of $\chi_{Z(G)}$ then $(G, Z(G), \lambda)$ is fully ramified. It is well known (see Lemma 4.3 of $[3])$ that if $(G, N, \theta)$ is fully ramified then there exists a group $G_{1}$ of central type and $\lambda \in \operatorname{Irr}\left(Z\left(G_{1}\right)\right)$ such that $(G, N, \theta)$ and $\left(G_{1}, Z\left(G_{1}\right), \lambda\right)$ are isomorphic character triples (see [5, p. 187] for the definition).

In $[\mathbf{3}]$ Howlett and Isaacs introduced the concept of an irreducible group of central type. We recall that definition: Let $G$ be a group of central type with $\lambda \in \operatorname{Irr}(Z(G))$ fully ramified in $G$. Then $G$ is reducible if $\lambda$ is fully ramified in some normal subgroup $N$ of $G$ with $Z(G)<N<G$. Otherwise, $G$ is irreducible.

In [2] Gagola raised the question about the nilpotence of irreducible groups of central type. The answer is negative as Example 8.2 of [3] shows. Our result is the following

THEOREM. Let $G$ be an irreducible group of central type, $Z=Z(G)$ and $\bar{G}=$ $G / Z$. Let $\lambda \in \operatorname{Irr}(Z(G))$ be fully ramified in $G$. Then

(i) If there exists a prime $p$ such that $|\operatorname{socle}(\bar{G})|_{p}>\mid \bar{G}$ : $\left.\operatorname{socle}(\bar{G})\right|_{p}$ then $G$ is nilpotent.

(ii) $F(\bar{G} / \operatorname{socle}(\bar{G}))=F(\bar{G}) / \operatorname{socle}(\bar{G})$, where $F(X)$ denotes the Fitting subgroup of $X$. As a consequence, if $\bar{G} / \operatorname{socle}(\bar{G})$ has a (nontrivial) normal Sylow subgroup then $G$ is nilpotent.

We need the following

LEMMA. Let $G$ be an irreducible group of central type, $Z=Z(G)$ and $\bar{G}=G / Z$. Let $\lambda \in \operatorname{Irr}(Z(G))$ be fully ramified in $G$. Then, assuming that $G$ is not nilpotent, we have that $\lambda$ is extendible to the preimage of $\operatorname{socle}(\bar{G})$ in $G$.

Received by the editors December 14, 1984 and, in revised form, May 1, 1985.

1980 Mathematics Subject Classification. Primary 20C15. 
ProOF OF THE LEMMA. The group $G$ is solvable by Theorem 7.3 of $[3]$. We may assume that $\lambda$ is faithful.

First we show that $\lambda$ is extendible to the preimage $A$ in $G$ of any minimal normal subgroup $\bar{A}$ of $\bar{G}$. As $\bar{G}$ is solvable, $\bar{A}$ is an elementary abelian group. Then there exists a subgroup $A_{1}$ normal in $G$ with $Z \leq A_{1} \leq A$ such that $\lambda$ is extendible to $A_{1}$ and $\left(A, A_{1}, \psi\right)$ is fully ramified for any $\psi \in \operatorname{Irr}\left(A_{1} \mid \lambda\right)$ (see [4, Theorem 2.7]). As $G$ is irreducible and $\bar{A}$ is a chief factor of $\bar{G}$, we have $A=A_{1}$ and our claim is verified.

Let $X$ be the preimage in $G$ of $\operatorname{socle}(\bar{G})$. Take $\bar{A}_{1}$ and $\bar{A}_{2}$ minimal normal subgroups of $G$ such that $\left|\bar{A}_{1}\right| \leq\left|\bar{A}_{2}\right|$. Let $\chi$ be the unique irreducible constituent of $\lambda^{G}$. If $\psi$ is an extension of $\lambda$ to $A_{1}$, then $\operatorname{Irr}\left(A_{1} \mid \lambda\right)=\left\{\psi \mu \mid \psi \in \operatorname{Irr}\left(A_{1} / Z\right)\right\}$. But $\operatorname{Irr}\left(A_{1} \mid \lambda\right)$ is also the set of irreducible constituents of $\chi_{A_{1}}$ and hence $G$ permutes its elements transitively by conjugation.

As socle $(\bar{G})$ is abelian then $I_{A_{2}}(\psi)=I_{A_{2}}(\psi \mu)$ for every $\mu$ and hence $I_{A_{2}}(\psi)$ is normal in $G$. Thus $I_{A_{2}}(\psi)=A_{2}$ or $I_{A_{2}}(\psi)=Z$. In the second case the character $\psi^{A_{1} A_{2}}$ is irreducible and its degree is $\left|\bar{A}_{2}\right|$. Hence $\left|\bar{A}_{2}\right|^{2} \leq\left|A_{1} A_{2}: Z\left(A_{1} A_{2}\right)\right|$. As $Z$ is central in $A_{1} A_{2}$ this forces to $\left|\bar{A}_{1}\right|=\left|\bar{A}_{2}\right|$ and thus $\left(A_{1} A_{2}, Z, \lambda\right)$ is fully ramified. As $A_{1} A_{2}$ is a normal nilpotent subgroup of $G$ this contradicts the irreducibility of $G$. Hence $A_{2}$ leaves invariant each $\psi \mu$ and, as $\psi \mu$ is linear, we have

$$
\left[A_{1}, A_{2}\right] \leq \bigcap_{\mu} \operatorname{Ker}(\psi \mu)=\operatorname{Ker} \lambda^{A_{1}}=1
$$

since $\lambda$ is faithful.

Now $X$ is abelian and clearly $\lambda$ is extendible to $X$.

ProOF of THE TheOREM. As in the Lemma, $G$ is solvable and $\lambda$ is faithful.

(i) Let $X$ be the preimage of $\operatorname{socle}(\bar{G})$ in $G$. If $G$ is not nilpotent then $\lambda$ is extendible to $X$ by the Lemma. Take a prime $p$ satisfying the hypothesis and let $P \in S_{p}(G)$. Put $\lambda=\prod_{q} \lambda_{q}$ where $\lambda_{q} \in \operatorname{Irr}\left(Z_{q}\right), Z_{q} \in S_{q}(Z)$. Now $\left(P, Z(P), \lambda_{p}\right)$ is fully ramified and $Z(P)=Z \cap P$ (see $\left[\mathbf{1}\right.$, Theorem 2]). Let $\chi_{p}$ be the unique irreducible constituent of $\lambda_{p}^{P}$. Let $\psi_{p}$ be an extension of $\lambda_{p}$ to $X \cap P$. Now $\chi_{p}$ is a constituent of $\psi_{p}^{P}$ and $\chi_{p}(1)^{2} \leq|P: X \cap P|^{2}=|\bar{G}: \operatorname{socle}(\bar{G})|_{p}^{2}<|\bar{G}|_{p}=|P: Z(P)|$, a contradiction.

(ii) Put $\lambda=\prod_{q} \lambda_{q}$, where $\lambda_{q} \in \operatorname{Irr}\left(Z_{q}\right), Z_{q} \in S_{q}(Z)$. If $Q \in S_{q}(G)$ then $Z(Q)=$ $Q \cap Z$ and $\left(Q, Z(Q), \lambda_{q}\right)$ is fully ramified as in (i). Let $\bar{X}_{q} \in S_{q}(\bar{X}), q$ a prime. If $\chi_{q}$ is the unique irreducible constituent of $\lambda_{q}^{Q}$ then the elements of $\operatorname{Irr}\left(X_{q} \cap Q \mid \lambda_{q}\right)$ are the irreducible constituents of $\left(\chi_{q}\right)_{X_{q} \cap Q}$. Hence they are conjugate in $Q$. But $\lambda_{q}$ is invariant in $G$ and $X_{q} \cap Q$ is normal in $G$. Hence $G$ permutes by conjugation the elements of $\operatorname{Irr}\left(X_{q} \cap Q \mid \lambda_{q}\right)$. We conclude that if $\psi_{q} \in \operatorname{Irr}\left(X_{q} \cap Q \mid \lambda_{q}\right)$ then $I_{G}\left(\psi_{q}\right)$ contains a Hall $q^{\prime}$-subgroup of $G$.

We may assume that $G$ is not nilpotent and then the Lemma applies. Hence the elements of $\operatorname{Irr}\left(X_{q} \cap Q \mid \lambda_{q}\right)$ are linear and $X$ is abelian as we showed. Now let $T$ be a subgroup of $G$ such that $\bar{T} / \bar{X}$ is a normal $p$-subgroup of $\bar{G} / \bar{X}$ for a prime $p$. We show that $O_{p^{\prime}}(\bar{X}) \leq Z(\bar{T})$. Take $q \neq p$ and $\bar{X}_{q} \in S_{q}(\bar{X})$. As $X$ is abelian and $T / X$ is a normal $q^{\prime}$-subgroup of $G / X$, we have that $T$ leaves invariant each element of $\operatorname{Irr}\left(X_{q} \cap Q \mid \lambda_{q}\right)$ by the preceding paragraph. Thus $\left[T, X_{q} \cap Q\right] \leq \operatorname{Ker} \lambda_{q}^{X_{q} \cap Q}=1$ since $\lambda_{q}$ is faithful. Thus our claim is verified and hence $\bar{T}$ is nilpotent. Then $F(\bar{G} /$ socle $(\bar{G})) \leq F(\bar{G}) /$ socle $(\bar{G})$. As the reverse inclusion is obvious, the first part 
of (ii) is verified. To prove the second take a prime $p$ and that $\bar{G} / \operatorname{socle}(\bar{G})$ is $p$ closed and let $P$ be a Sylow $p$-subgroup of $G$. Now $P$ is normal in $G$. As $(P Z, Z, \lambda)$ is fully ramified, we deduce that $G$ is nilpotent.

We recall the following definition (see [2, p. 123]): A p-group $Q$ is reductive if, for every fully ramified triple $(H, Z, \lambda)$ with $Q$ isomorphic to a Sylow $p$-subgroup of $H / Z$, the irreducible constituent $\chi$ of $\lambda^{H}$ is fully ramified in $O^{p}(H) Z$.

In Lemma 4.4 of [2] Gagola showed that every $p$-group of order $p^{4}$ is reductive. There exist nonreductive $p$-groups at least for $p=2,3$, as Example 8.2 of $[3]$ shows. As this example is really complicated and it is not apparent that it might be extended to the remaining primes, we construct a nonreductive $p$-group of order $p^{6}$ for $p$ odd.

We consider $A_{0}=\langle a, b, c, z\rangle \simeq\left(C_{p}\right)^{4}$. By the theory of cyclic extensions there exists an extension $A$ of $A_{0}$ by $B_{0}=\langle\alpha, \beta, \gamma\rangle \simeq\left(C_{p}\right)^{3}$ where $\alpha^{p}=\beta^{p}=\gamma^{p}=$ $1,[\alpha, \beta]=c,[\alpha, \gamma]=a,[\beta, \gamma]=b,[a, \alpha]=[b, \beta]^{-1}=[c, \gamma]=z$, the remaining commutators being trivial.

Let $B$ be the quaternion group of order 8 acting on $A$ as follows: If $e$ and $f$ are generators of $B$ then $\alpha^{f}=\beta, \beta^{f}=\alpha, \gamma^{f}=\gamma^{-1}, a^{f}=b^{-1}, b^{f}=a^{-1}, c^{f}=$ $c^{-1}, z^{f}=z$ and $e$ acts trivially.

It is an easy check that this action is well defined. Let $G$ be the natural semidirect product of $A$ by $B$. Let $\lambda$ be a nonprincipal character of $A_{0} /\langle a, b, c\rangle$ and $\mu$ a faithful character of $\langle e\rangle$. Consider $\lambda \times \mu$ as a character of $A_{0} \times\langle e\rangle$. Clearly $I_{G}(\lambda \times \mu)=$ $I_{G}(\lambda) \cap I_{G}(\mu)$. It is an easy check that $N_{A}(\operatorname{Ker} \lambda)=A_{0}$ and hence $I_{A}(\lambda)=A_{0}$. Clearly $I_{B}(\mu)=\langle e\rangle$. Then $I_{G}(\lambda \times \mu)=A_{0} \times\langle e\rangle$. Thus $(\lambda \times \mu)^{G}$ is irreducible and $(\lambda \times \mu)^{G}(1)^{2}=|G: Z(G)|$. Hence $G$ is of central type. Now $O^{p}(G)$ contains $\alpha^{-1} \beta, \gamma$ and $c$. As is normal in $G$, it contains $[\alpha, \gamma]$ and $[\beta, \gamma]$. Thus $\left|G: O^{p}(G) Z\right|=p$ and $(\lambda \times \mu)^{G}$ is not fully ramified in $O^{p}(G) Z$.

Using a similar technique it is possible to construct a nonreductive 2-group of order $2^{6}$.

\section{REFERENCES}

1. F. De Meyer and G. J. Janusz, Finite groups with an irreducible representation of large degree, Math. Z. 108 (1969), 145-153.

2. S. M. Gagola, Characters fully ramified over a normal subgroup, Pacific J. Math. 55 (1974), 107-126.

3. R. B. Howlett and I. M. Isaacs, On groups of central type, Math. Z. 179 (1982), 555-569.

4. I. M. Isaacs, Characters of solvable and symplectic groups, Amer. J. Math. 95 (1973), 594-635.

5. __ Character theory of finite groups, Academic Press, New York, 1976.

Departamento de Algebra, Facultad de Ciencias, Universidad de Zaragoza, 50009 ZARAGOZA, SPAIN 